4. "Ein hoher Gehalt der Lösung an $\mathrm{Kupferchlorid} \mathrm{ist} \mathrm{sowohl} \mathrm{für} \mathrm{die} \mathrm{Stromausbeute}$ ungünstig, als auch für die Reinbeit des Kupfers, indem die Chlorürbildung hierdurch vermehrt wird. Macht man aber den Gehalt an Kupferchlorid allzu gering (bei unseren Versuchen bei etwa $0,05 \mathrm{Mol}$ pro Liter), so wird das ausgeschiedene Kupfer schwammig."

5. „Temperaturerhöhung wirkt sowohl ungünstig auf die Stromausbeute, als auf die Reinheit des Kupfers, doch darf man auch nicht zu tief dabei gehen, da sonst das Kupfer schwammig wird."
6. "Vermehrung der Stromdichte lässt die Stromausbeute steigen, da die Löslichkeit des Kupfers in dem Elektrolyten nicht wesentlich davon abhängt. Dies gilt so lange, als das Farad a y sche Gesetz für Kupfer noch annähernd erfüllt ist; bei höheren Stromdichten beginnt dann die Stromausbeute zu sinken, indem Wasserstoff an der Kathode abgeschieden wird."

7. "Aus einem Elektrolyten von der $\mathrm{Zu}$ sammensetzung: o, $\mathrm{I} \mathrm{Mol} \mathrm{CuCl}_{2}$, o, $\mathrm{Mol} \mathrm{KCl}$ und o,4 Mol $\mathrm{NaCl}$ konnte ein Kupfernicderschlag erhalten werden von dem Reinheitsgrade 99,90 bis $99,98 \% \mathrm{Cu}^{\prime \prime}$.

\title{
MOLEKULARGEWICHTS-BESTIMMUNG.
}

Molekulargewichtsbestimmungen flüssiger Stoffe. W. Kistiakowski (Journ. d. Russ. phys.chem. Ges. 34, 70-90, 1902). Verf. bezweckt mit diescr Arbeit eine theoretische und experimentelle Vereinfachung des bekannten Ramsayschen Verfahrens zur Bestimmung der Molekulargewichte flüssiger Stoffe aus den Messungen ihrer Oberflächenenergie. Was aber seine theoretischen Auseinandersetzungen anbetrifft, so stellen dieselben eine Wiedergabe der Eötvösschen Ansichten dar, indem auf dessen Ausdruck: zurückgegriffen wird.

$$
\frac{r_{1} v_{1}^{2 / 3}}{T_{1}}=\frac{r_{2} v_{2}^{2 / 3}}{T_{2}}
$$

Durch entsprechende Umrechnung der Ramsayschen Experimentaldaten wird vom Verfasser gezeigt, dass der oben genannte Ausdruck

$$
\frac{r^{2 / 3}}{T}=\mathrm{Rm}
$$

den er als "Kapazität für Oberflächenenergie" bezeichnet, für nichtassociierte Stoffe bei korrespondierenden Temperaturen oder Drucken eine konstante Grösse darstellt, wie aus folgenden

\begin{tabular}{|c|c|c|c|}
\hline \multicolumn{4}{|c|}{$\operatorname{Rm}$ für $\frac{T}{T_{k}}=0,6_{4}$} \\
\hline$C_{i j} H_{i j} \mathrm{Cl}$ & . I, I4 & $\mathrm{HCOOCH}_{3}$ & I, I I \\
\hline$C_{6} H_{0}$ & $x, x_{5}$ & $H C O O C_{2} H_{5}$ & $\mathrm{Y}, \mathbf{I} \mathbf{I}$ \\
\hline $\mathrm{CCl}_{4}$. & $\mathrm{I}, \mathrm{I} 5$ & $\mathrm{CH}_{\mathrm{i}} \mathrm{COOC \textrm {CH } _ { 3 }}$ & $I, 16$ \\
\hline$(\mathrm{C}, \mathrm{H}, \mathrm{O}$ & $\mathrm{I}, \mathrm{I} 7$ & $\mathrm{CH}_{3} \mathrm{COOC}_{2} \mathrm{H}_{3}$ & $\mathrm{I}, \mathrm{I} 8$ \\
\hline \multicolumn{4}{|c|}{$\mathrm{Rm}$ für $\frac{P}{P_{k}}=\mathrm{o}, 0224 \mathrm{I}$} \\
\hline $\mathrm{C}_{\mathrm{i}} \mathrm{H}_{\mathrm{i}} \mathrm{Cl}$ & 1,16 & $\mathrm{CH}_{3} \mathrm{COO} \mathrm{C}_{2}$ & $\mathrm{I}, \mathrm{II}$ \\
\hline$C_{63} H_{6}$ & $I, 18$ & $\mathrm{C}_{2} \mathrm{H}_{5} \mathrm{COOC \textrm {CH } _ { 3 }}$ & $\mathrm{I}, \mathrm{II}$ \\
\hline $\mathrm{CCl}_{4}$ & . I, II & $\mathrm{CH}_{3} \mathrm{COOC}_{3} \mathrm{H}_{7}$ & 1, so \\
\hline & . $\mathrm{J}, \mathrm{TI}$ & 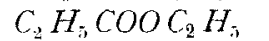 & $\mathrm{r} \mathrm{IL}$ \\
\hline & 1,09 & & $\mathrm{I}, \mathrm{I} 2$ \\
\hline & 1,10 & i. $\mathrm{C}_{3} \mathrm{H}_{7} \mathrm{COOCH}_{3}$ & $\mathrm{I}, \mathrm{I} 3$ \\
\hline
\end{tabular}
Tabellen zu ersehen ist:

Ohne auf die Frage einzugehen, weshalb Ramsay (Zeitschr. f. phys. Chem. 12, 437) seiner Zeit zu einem entgegengesetzten Ergebnis bezüglich der Konstanz des Ausdrucks (I) für korrespondierende Temperaturen gekommen war, betrachtet Verf. die Konstanz desselben als

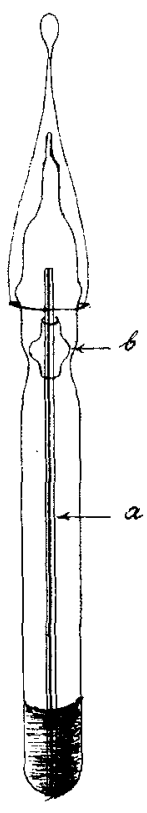

Fig. I37.

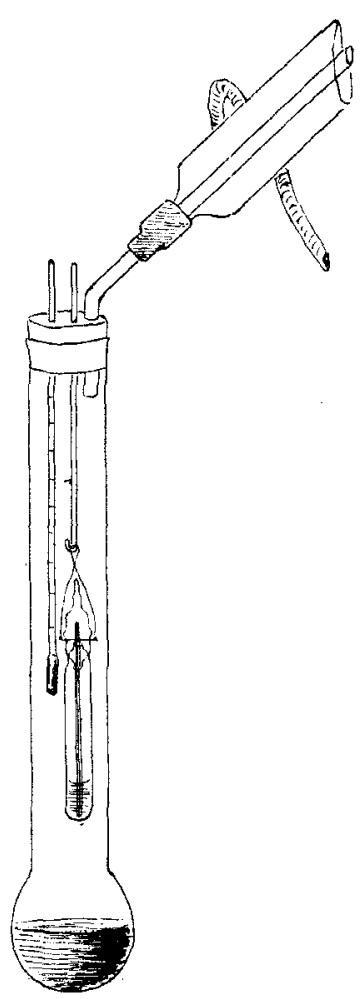

Fig. 138 . erwiesen und leitet daraus folgende Formel:

$$
M G=p\left(\frac{\mathrm{Rm} \cdot T}{\gamma}\right)^{3 / 2}
$$

für das Molekulargewicht flüssiger Stoffe ab. Die allgemeine Gültigkeit dieser letzteren soll durch eine "gute" Uebereinstimmung der mit ihrer Hilfe berechneten Molekulargewichte mit den "theoretischen" an etwa fünfzig nicht associierten Flüssigkeiten dargethan werden. 
Die vom Verf. vorgeschlagene Abänderung des Ramsayschen Verfahrens zur Messung kapillarer Steighöhen ist aus den angeführten Fig. I 37 und $13^{8}$ ersichtlich. Zwecks Centrierung der Kapillare $a$ wird auf dieselbe ein kurzes Rohrstück $b$ mit drei Vorsprüngen aufgesetzt. Die Ablesung der beiden Menisken geschieht mittels eines Kathetometers. Das Wesentliche der „Vereinfachung" der Messmethode besteht aber darin, dass man den Durchmesser der Kapillare nicht direkt zu bestimmen braucht, sondern ihn aus den Beobachtungen der kapillaren Steighöhen des Benzols unter Zugrundelegung von Ramsays Daten berechnen kann, und mit diesem "scheinbaren Durchmesser" weiter operiert.
Auf diese Weise werden folgende Daten ermittelt:

\begin{tabular}{|c|c|c|c|c|c|}
\hline \multirow[b]{2}{*}{ Für } & & $100 \alpha^{2}$ & \multicolumn{3}{|c|}{ Molekulargewicht } \\
\hline & $\begin{array}{c}\text { Tem- } \\
\text { peratur }\end{array}$ & $\begin{array}{l}\text { beim } \\
\text { Siede } \\
\text { punist }\end{array}$ & $\begin{array}{l}\text { nachder } \\
\text { Siede- } \\
\text { temp. }\end{array}$ & $\begin{array}{c}\text { bei } \\
\text { korresp. } \\
\text { Temp. }\end{array}$ & 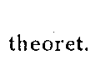 \\
\hline I. Zinutetr & - & 1,76 & 264 & $2+2$ & 260 \\
\hline 2. Allylbromid. & - & $3, \mathrm{I}_{4}$ & I. 3 & - & $\mathrm{I} 2 \mathrm{I}$ \\
\hline 3. Aethylbenzol & - & 4,43 & - & ro2 & 106 \\
\hline 4. Isodibutylen & 278 & 3,90 & I 35 & ror & $\mathrm{TI2}$ \\
\hline 5. Caprylen . & - & 3.90 & $\mathrm{I}_{40}$ & 98 & II2 \\
\hline $\begin{array}{l}\text { 6. Vinyltri- } \\
\text { methylen }\end{array}$ & 223 & 2,54 & 66,5 & - & 68,0 \\
\hline
\end{tabular}

J. von Zawidski.

\section{ZUR THEORIE DER LÖSUNGEN.}

Kryoskopische Untersuchungen in anorganischen Lösungsmitteln. St. Tolloc zko (Bull. de l'Acad. des Sciences de Cracovie Igor, I-22). Im Jahre I 899 hat der Verf. gezeigt, dass Salze in geschmolzenem Antimonchlorür $\mathrm{Sb} \mathrm{Cl}_{3}$, zu niedrige Molekulargewichte aufweisen, was im Sinne der Dissociationstheorie auf einen Zerfall in Ionen schliessen liess. Die vorliegende Untersuchung bringt die Erweiterung des Thatsachenmaterials sowohl auf das oben erwähnte Lösungsmittel, wie auch auf Antimonbromür, $\mathrm{Sb} \mathrm{Br}_{3}$, Arsenbromür, $\mathrm{As} \mathrm{Br}_{3}$, und Zinnbromid, $\mathrm{Sn} \mathrm{Br}_{4}$. Es ergaben sich für die molekulare Gefrierpunktserniedrigung folgende Werte:

$\begin{array}{lcc} & E \text { kryoskop. } & \text { Ekalorimetr. } \\ \mathrm{SbCl} & 184 & 177 \\ \mathrm{Sb} \mathrm{Br} & 267 & 274 \\ \mathrm{As} \mathrm{Br}_{3} & 206 & - \\ \mathrm{Sn} \mathrm{Br}_{4} & 305 & 259\end{array}$

Wie ersichtlich, ergiebt sich aus kryoskopischen Messungen an Nichtelektrolyten ein Wert für die Konstante $E$, welcher dem nach der van't Hoffschen Formel berechneten genügend nahe kommt. Ganz anders verhalten sich Haloïde von mehr oder weniger salzartigem Verhalten, wie $\mathrm{KCl}, \mathrm{HgCl}_{2}, \mathrm{AsCl}_{3}, \mathrm{As} \mathrm{Br}_{3}$ u. a. So ergeben Kaliumchlorid und Quecksilberchlorid in Antimonchlorür Werte für die Konstante $E$, welche - in verdünnten Lösungen - bedeutend höher als der normale sind. Hier ist also eine elektrolytische Dissociation wahrscheinlich. Der Dissociationsgrad für $\mathrm{KCl}$ beträgt für eine Konzentration von $\circ$, oor $\mathrm{Mol}$ in $\mathrm{I}$ oo $\mathrm{g}=0,56$. Komplizierter liegen jedoch die Verhältnisse bei den Chloriden, Bromiden und Jodiden der Elemente $A s, S b, B i, S n$. Hier findet man sowohl höhere als niedrigere $E$-Werte, $\mathrm{ja}$, in einigen Fällen ist die Konstante sogar negativ, d.h. der Gefrierpunkt wird durch Zusatz des gelösten Körpers erhöht. Diese Verhältnisse werden sachgemäss dadurch erklärt, dass im ersten Falle eine chemische Wechselwirkung zwischen dem gelösten Stoff und dem Lösungsmittel, im zweiten Fall, wenn die Konstante zu niedrig ist, Bildung von festen Lösungen und isomorphen Gemischen angenommen wird. (Der Ref. möchte noch auf eine dritte Möglichkeit hinweisen, nämlich dass der kryohydratische Punkt in der Nähe des Gefrierpunktes des Lösungsmittels liegt; dieser Fall dürfte bei der Erhöhung des Gefrierpunktes vorliegen; analoges Beispiel liefern Gemenge einiger optischen Antipoden. Die Untersuchung der Zusammensetzung der festen Phase dürfte deshalb ein hohes Interesse beanspruchen.)

Dicse Fälle zeigen aber auch zu gleicher Zeit, wie vorsichtig man in der Interpretation der kryoskopischen Messungen in Bezug auf etwa vorhandene Dissociation sein muss. Es sei hier deshalb erwähnt, dass auf Grund von Leitfähigkeitsmessungen - welche zu bedeutend sicherern Schlüssen im allgemeinen führen - Walden (Zeitschr., f. anorgan. Chem. 25, 219; 29, 37I) ganz unzweideutig nachgewiesen hat, dass das Antimontrichlorid solche Stoffe wie Kaliumbromid thatsächlich zu ionisieren vermag, dagegen dem Arsenbromid diese Eigenschaft vollständig abgeht, und damit diese Verhältnisse auf eine vollkommen sichere Grundlage gestellt hat.

$$
\text { M. Centnerszwer. }
$$

Ueber die Dissociation des Chloralhydrates und Chloralalkoholates in Lösungen. $\mathrm{L}$. $\mathrm{Br}$ uner (Bull. de l'Acad. des Sciences de Cracovie I gor, 464-473). Der Verf. hat sich die Frage vorgelegt, ob die nicht-elektrolytische Dissociation solcher Stoffe, welche im Dampfzustand dissociiert sind, auch in Lösungen stattfindet. Als Objekte dienten Cbloralhydrat $\left(\mathrm{CCl}_{3} \cdot \mathrm{CH}(\mathrm{OH})_{2}\right)$ und Chloralalkoholat $\left(\mathrm{CCl}_{3} \cdot \mathrm{CHO} \cdot \mathrm{C}_{2} \mathrm{H}_{5} \mathrm{OH}\right)$. Ebullio- 